\title{
Posttreatment PET/CT Rather Than Interim PET/CT Using Deauville Criteria Predicts Outcome in Pediatric Hodgkin Lymphoma: A Prospective Study Comparing PET/CT with Conventional Imaging
}

Sameer Bakhshi ${ }^{1}$, Sainath Bhethanabhotla ${ }^{1}$, Rakesh Kumar ${ }^{2}$, Krishankant Agarwal2 ${ }^{2}$ Punit Sharma ${ }^{2}$, Sanjay Thulkar ${ }^{3}$, Arun Malhotra $^{2}$, Deepa Dhawan ${ }^{1}$, and Sreenivas Vishnubhatla ${ }^{4}$

${ }^{1}$ Department of Medical Oncology, Dr. B. R.A. Institute Rotary Cancer Hospital, All India Institute of Medical Sciences, New Delhi, India; ${ }^{2}$ Department of Nuclear Medicine, All India Institute of Medical Sciences, New Delhi, India; ${ }^{3}$ Department of Radiology, Dr. B. R.A. Institute Rotary Cancer Hospital, All India Institute of Medical Sciences, New Delhi, India; and ${ }^{4}$ Department of Biostatistics, All India Institute of Medical Sciences, New Delhi, India

Data about the significance of ${ }^{18} \mathrm{~F}-\mathrm{FDG}$ PET at interim assessment and end of treatment in pediatric Hodgkin lymphoma $(\mathrm{HL})$ are limited. Methods: Patients ( $\leq 18 \mathrm{y}$ ) with HL were prospectively evaluated with contrast-enhanced CT (CECT) and PET combined with low-dose CT (PET/CT) at baseline, after 2 cycles of chemotherapy, and after completion of treatment. Revised International Working Group (RIW) criteria and Deauville 5 point-scale for response assessment by PET/CT were used. All patients received doxorubicin (Adriamycin), bleomycin, vinblastine, dacarbazine chemotherapy along with involved-field radiotherapy (25 Gy) for early stage (IA, IB, and IIA) and advanced stage (IIB-IV) with bulky disease. Results: Of the 57 enrolled patients, median follow-up was $81.6 \mathrm{mo}$ (range, 11-97.5 mo). Treatment decisions were based on CECT. At baseline, PET/CT versus CECT identified 67 more disease sites; 23 patients $(40.3 \%)$ were upstaged and of them in 9 patients (39\%) upstaging would have affected treatment decision; notably none of these patients relapsed. The specificity of interim PET/ CT based on RIW criteria (61.5\%) and Deauville criteria (91.4\%) for predicting relapse was higher than CECT (40.3\%) $(P=0.03$ and $P<$ 0.0001 , respectively). Event-free survival based on interim PET/CT (RIW) response was $93.3 \pm 4.1$ versus $89.6 \pm 3.8$ (positive vs. negative scan, respectively; $P=0.44$ ). The specificity of posttreatment PET/CT (Deauville) was $95.7 \%$ versus $76.4 \%$ by CECT $(P=0.006)$. Posttreatment PET/CT (Deauville) showed significantly inferior overall survival in patients with positive scan versus negative scan results ( $66.4 \pm 22.5$ vs. $94.5 \pm 2.0, P=0.029$ ). Conclusion: Interim PET/CT has better specificity, and use of Deauville criteria further improves it. Escalation of therapy based on interim PET in pediatric HL needs further conclusive evidence to justify its use. Posttreatment PET/CT (Deauville) predicts overall survival and has better specificity in comparison to conventional imaging.

Key Words: PET-CT; pediatric Hodgkin lymphoma; CECT

J Nucl Med 2017; 58:577-583

DOI: 10.2967/jnumed.116.176511

Received Apr. 4, 2016; revision accepted Sep. 8, 2016

For correspondence or reprints contact: Sameer Bakhshi, Department of Medical Oncology, Dr. B. R.A. Institute Rotary Cancer Hospital, All India Institute of Medical Sciences, New Delhi-110 029, India.

E-mail: sambakh@hotmail.com

Published online Oct. 6, 2016.

COPYRIGHT (C) 2017 by the Society of Nuclear Medicine and Molecular Imaging.
$\mathbf{P}$ ediatric Hodgkin lymphoma (HL) is a malignancy with high cure rates with currently available combined-modality treatment (chemotherapy and radiotherapy) (1). The current emphasis is to identify patients with high risk of relapse and to minimize the long-term side effects in the survivors. A risk-adapted treatment approach based on interim response assessment with PET/CT is advocated in adult patients with HL for this purpose (2). Studies in adult patients with HL have shown that a positive interim PET/CT scan predicts higher chance of relapse and poor outcome $(3,4)$.

Contrast-enhanced CT (CECT) has been conventionally used for staging and response assessment for children with HL. It is an easily available, affordable, and reproducible investigation for staging and response assessment. The evidence for the role of PET/CT imaging in pediatric HL in evaluation, response assessment, and prognostic value is limited and predominantly retrospective in nature (5-9).

Two prospective studies in a small number of subjects have shown conflicting results. A study by Furth et al. (10) demonstrated that interim PET/CT had excellent negative predictive value (NPV) but a poor positive predictive value (PPV) for relapse. Another study, by Ilivitzki et al. (11), showed a higher PPV for interim PET/CT. Notably, the criteria for response assessment by PET/CT were different in both the studies. The prognostic impact of interim and posttreatment PET/CT on event free survival (EFS) and overall survival (OS) was also not reported in these studies. We prospectively studied the role of combined ${ }^{18} \mathrm{~F}$-FDG PET/CT compared with CECT alone in identifying patients with residual disease and its prognostic significance to predict relapse in pediatric HL.

\section{MATERIALS AND METHODS}

\section{Study Objective}

The primary objective was to evaluate the sensitivity, specificity, $\mathrm{PPV}$, and NPV of interim and posttreatment PET/CT as compared with CECT alone in pediatric HL patients for predicting relapse. The secondary objective was to determine the prognostic significance of a positive PET/CT result at interim assessment and after treatment on EFS and OS. 


\section{Study Design}

This was a prospective study in children with HL treated with a uniform protocol and compared at baseline, interim, and posttreatment scans of CECT and PET/CT.

\section{Participants}

We prospectively enrolled children (age $\leq 18$ y) diagnosed with HL attending the oncology clinic at our center from January 2008 to December 2010. The study was performed per the guidelines of Declaration of Helsinki, after approval by the Institute Ethics Committee. Written informed consent was taken from parents or child before enrollment into the study. All patients were enrolled after establishing tissue diagnosis. Other investigations apart from CECT and PET/CT included complete blood counts, erythrocyte sedimentation rate, serum lactate dehydrogenase levels, liver and renal function tests, and bone marrow aspiration and biopsy. Modified Ann Arbor classification was used for staging (12).

\section{Treatment and Response Assessment}

All patients were treated with standard doxorubicin, bleomycin, vinblastine, dacarbazine chemotherapy (13). Patients in early stage (stage $1 \mathrm{~A}, 1 \mathrm{~B}$, and $2 \mathrm{~A}$ ) received 4 cycles of chemotherapy. Patients with advanced stage (stage $2 \mathrm{~B}, 3$, and 4 ) received 6-8 cycles of chemotherapy. All early-stage patients and advanced-stage patients with bulky disease received low-dose involved-field radiotherapy ( 25 Gy in 15 fractions). Notably, all patients were treated on the basis of baseline CECT staging irrespective of PET/CT staging.

Patients underwent whole-body PET/CT and CECT of the neck, chest, abdomen, and pelvis at baseline for staging (PET-1 and CT-1), after 2 cycles of chemotherapy for interim assessment (PET-2 and CT2 ), and after completion of chemotherapy (PET-3 and CT-3). Scans were obtained at least $2 \mathrm{wk}$ after chemotherapy cycle.

\section{CECT Acquisition and Analysis}

CECT of the neck, chest, and abdomen was performed on a 64-slice MDCT (Definition AS; Siemens). All patients were given oral contrast (2\% iomeprol, $500-1,000 \mathrm{~mL}$ ) divided into 2 doses at 45 and $15 \mathrm{~min}$ before scanning. Intravenous nonionic contrast (iomeprol, $400 \mathrm{mg} / \mathrm{mL}$ ) was injected as a bolus before the scan at a dose of $2 \mathrm{~mL} / \mathrm{kg}$. Sections of $5 \mathrm{~mm}$ were taken from below the base of the skull up to the pelvic floor.

An experienced radiologist prospectively reviewed all CECT scans on a work station. Response was defined on CECT as complete response (CR), partial response (PR), stable disease, and progressive disease (PD) on the basis of the revised International Working Group (RIW) response criteria (14).

\section{F-FDG PET/CT Acquisition Protocol}

All patients received intravenous injection of 6-7 MBq/kg (minimum, $110 \mathrm{MBq}$; maximum, $370 \mathrm{MBq}$ ) of ${ }^{18} \mathrm{~F}-\mathrm{FDG}$. PET/CT imaging was performed after 45-60 min of injection. PET/CT images were acquired on a dedicated PET/CT scanner (Biograph 2; Siemens Medical Solutions). For the CT part of PET/CT, no oral or intravenous contrast agent was administered. CT was acquired with a spiral dualsection method with the following parameters: slice thickness of $4 \mathrm{~mm}$, pitch of 1 , matrix of $512 \times 512$ pixels, and pixel size of $1 \mathrm{~mm}$. After completion of CT, PET was performed in the same axial range with the patient in the same position. Three-dimensional PET was performed for 2-3 min per bed position, with a matrix of $128 \times 128$ pixels and slice thickness of $1.5 \mathrm{~mm}$. CT-based attenuation correction of PET emission images was performed. PET images were reconstructed by an iterative method with ordered-subset expectation maximization algorithm (2 iterations and 8 subsets). The reconstructed attenuation-corrected PET images, CT images, and fused PET/CT images were available for review in axial, coronal, and sagittal planes, along with maximum-intensity projections and 3-dimensional cine mode.

\section{${ }^{18}$ F-FDG PET/CT Image Analysis Using RIW \\ Response Criteria}

${ }^{18} \mathrm{~F}$-FDG PET/CT was evaluated by 2 nuclear medicine physicians (with 10 y experience in PET/CT imaging). Any positive findings on ${ }^{18}$ F-FDG PET were localized anatomically on nonenhanced CT. All patients were assessed for response as CR, PR, stable disease, and PD based on RIW response criteria (14).

The nuclear physicians were masked to the clinical details and radiologic response of patients.

\section{${ }^{18}$ F-FDG PET/CT Image Analysis Using Deauville Criteria (DC): Retrospectively Analyzed}

At the beginning of study, RIW response criteria were considered for image analyses. Later studies in adult patients with HL showed the predictive role of interim PET/CT assessment on survival using the Deauville 5-point scale (15). Thus, additionally, the PET/CT images of all patients were retrospectively reviewed by 2 experienced nuclear medicine physicians with more than 10 and 5 y experience in oncologic PET/CT imaging, respectively. Both of them were masked regarding patient details, outcomes, and CECT findings. Further, the 2 physicians independently reviewed the PET/CT images, and wherever there was discrepancy in findings a consensus was reached with mutual discussion.

For assessment of treatment response, the following definitions were used: CR, Deauville scores 1, 2, or 3 together with absence of ${ }^{18} \mathrm{~F}-\mathrm{FDG}$-avid bone marrow lesions irrespective of a persistent mass on CT; PR, Deauville score 4 or 5, but uptake is decreased compared with baseline and absence of structural progression on $\mathrm{CT}$; stable disease, no metabolic response, Deauville score 4 or 5 , with no significant change in ${ }^{18} \mathrm{~F}-\mathrm{FDG}$ uptake from baseline; and PD, Deauville score 4 or 5 with increasing intensity compared with baseline or any new ${ }^{18}$ F-FDG-avid lesions.

\section{Statistical Analysis}

SPSS software (version 16.0; SPSS Inc.) was used for data analyses. Sensitivity, specificity, PPV, and NPV were calculated using standard formulas. Kaplan-Meier survival analysis was used to determine EFS and OS. Data were censored on December 31, 2015. OS was calculated from the date of diagnosis to death due to any cause. EFS was calculated from the date of diagnosis to the date of relapse or death due to any cause. The McNemar test with continuity correction was used for comparison of proportion of negative findings with each imaging modality. The $\kappa$-statistic was used to measure degree of agreement between investigators for using DC for PET/CT images.

\section{RESULTS}

Fifty-seven consecutive patients with pediatric HL were enrolled in the study, and their baseline characteristics are shown in Table 1. Their staging by CECT and PET/CT, response, and final status are shown in Supplemental Table 1 (supplemental materials are available at http://jnm.snmjournals.org). Median follow-up of the cohort was 81.6 mo (range, 11-97.5 mo). One patient in CR was lost to follow-up after 18 mo (patient 31). Four patients relapsed during follow-up, of whom 2 are currently doing well and are disease free after salvage therapy (patients 30 and 36). There were 3 deaths -2 patients died because of disease progression (patients 17 and 35) and 1 patient died of treatmentrelated pulmonary toxicity (patient 57) (Supplemental Table 1). The mean interval between CT and PET/CT was $10.8 \mathrm{~d}$ (median, $10 \mathrm{~d}$; range, 0-26 d) at baseline, $4 \mathrm{~d}$ (median, $2 \mathrm{~d}$; range, 0-20 d) at interim assessment, and $6 \mathrm{~d}$ (median, $3 \mathrm{~d}$; range, 0-26 d) at the end of treatment. 
TABLE 1

Baseline Characteristics

\begin{tabular}{lc}
\hline Characteristic $(n=57)$ & $n(\%)$ \\
\hline Sex & $42(73.6 \%)$ \\
\hline Male & $15(26.4 \%)$ \\
\hline Female & $10($ range, $4-18)$ \\
\hline Median age $(y)$ & \\
\hline Stage & $26(45.6 \%)$ \\
\hline Early stage (IA, IB, and IIA) & $31(54.4 \%)$ \\
\hline Advanced stage (IIB-IV) & $23(40.3 \%)$ \\
\hline Bulky disease & $81.6($ range, $11-97.5)$ \\
\hline Median follow-up (mo) & $32(56.1 \%)$ \\
\hline Subtype & $16(28.1 \%)$ \\
\hline Mixed cellularity & $3(5.2 \%)$ \\
\hline Nodular sclerosis & $1(1.7 \%)$ \\
\hline Lymphocyte rich & $1(1.7 \%)$ \\
\hline Lymphocyte depleted & $4(7.2 \%)$ \\
\hline NLPHL & $37(64.9)$ \\
\hline Unspecified & \\
\hline Involved-field radiotherapy & \\
\hline NLPHL $=$ nodular lymphocyte predominant Hodgkin lymphoma. \\
\hline
\end{tabular}

\section{Baseline Imaging}

A total of 219 disease sites were detected with PET/CT versus 152 sites with CECT scanning. Sixty-seven more disease sites were detected in 29 patients with baseline PET/CT whereas PET/ CT showed 4 fewer disease sites in 3 patients. Overall, 23 (40.3\%) patients were upstaged whereas $4(7 \%)$ were downstaged per PET/ CT results when compared with the baseline staging by CECT. Of the 23 patients who were upstaged on PET/CT, the upstaging would have affected treatment decision in $9(39 \%)$ patients.

Bone marrow and bone involvement were detected by PET/CT at baseline in 6 and 12 patients, respectively. Bone marrow biopsy, however, could confirm disease involvement in only 2 patients.

\section{Impact of Change of Baseline Staging by PET/CT on Outcome}

Of 23 patients who were upstaged by baseline PET/CT baseline, 1 patient relapsed (patient 35). However, in this patient the upstaging would not have changed the initial planned treatment per CT imaging.

\section{Interim Response Assessment (PET-2 [RIW] vs. CT-2)}

Interim response assessment was done in all patients. The sensitivity and specificity of positive PET-2 for predicting relapse was $25 \%$ and $61.5 \%$, respectively (Table 2). There was a significant difference between the number of patients who had a CR on PET-2 (RIW) versus CT-2 (36 [63.1\%] vs. 23 [40.3\%]; $P=$ 0.012). The specificity of positive PET-2 (RIW) for relapse was significantly better than CT-2 (61.5 vs. $40.3, P=0.03)$. However, the NPV at PET-2 (RIW) was similar to CT-2 (91.4\% vs. $95.4 \%$, $P=0.39$ ). Both the modalities had a low PPV for relapse.

The difference in EFS and OS of patients with positive and negative PET-2 scans (Figs. 1A and 1B) or achievement of CR on CT-2 at interim assessment was not statistically significant (Figs. 1C and 1D) (Table 3).

\section{Posttreatment Response Assessment (PET-3 [RIW] vs. CT-3)}

Fifty-five patients were tested with both modalities at treatment completion. Posttreatment PET-3 and CT-3 could not be done in 2 and 1 patient, respectively. There was no significant difference in the proportion of patients attaining CR in PET-3 and CT-3 $83.9 \%$ vs. $75 \%, P=0.24)$. The sensitivity and specificity of PET-3 (RIW) was $25 \%$ and $88 \%$, respectively (Table 4). No statistically significant differences in sensitivity, specificity, PPV, and NPV were observed between PET-3 (RIW) and CT-3 (Table 4).

At the end of treatment, PET-3 (RIW) was positive in 7 patients (4 patients had PD and 3 patients had PR). Of the 4 patients with PD in PET-3, 1 had primary refractory disease, 2 had false-positive (one patient had normal findings on fine-needle aspiration cytology of involved lymph node, and in the other patient image-guided cytology was not feasible but on subsequent imaging

TABLE 2

Interim Assessment

\begin{tabular}{|c|c|c|c|c|c|c|c|c|}
\hline \multirow[b]{2}{*}{ Parameter } & \multicolumn{2}{|c|}{ CT-2 $(n=56)$} & \multicolumn{2}{|c|}{ PET-2 (RIW) $(n=56)$} & \multirow[b]{2}{*}{$P^{\star}$} & \multicolumn{2}{|c|}{ PET-2 (DC) $(n=52)$} & \multirow[b]{2}{*}{$P^{\dagger}$} \\
\hline & CR & No CR & Positive & Negative & & Positive & Negative & \\
\hline Relapse $(n=4)$ & 1 & 3 & 1 & 3 & & 0 & 4 & \\
\hline Remission $(n=52)^{\ddagger}$ & 21 & 31 & 20 & 32 & & 4 & 44 & \\
\hline Sensitivity (\%) & 75 (21.9-98.6) & & $25(13.1-78)$ & & 0.18 & $0(0-60.4)$ & & 0.04 \\
\hline Specificity (\%) & $40.3(27.3-54.8)$ & & $61.5(47-74.3)$ & & 0.03 & 91.4 (78.7-97.2) & & $<0.0001$ \\
\hline PPV (\%) & $8.8(2.3-24.8)$ & & $4.7(0.2-25.8)$ & & 0.57 & $0(0-60.4)$ & & 0.54 \\
\hline NPV (\%) & $95.4(75.1-99.7)$ & & 91.4 (75.8-97.7) & & 0.39 & 91.4 (78.7-97.2) & & 0.4 \\
\hline $\begin{array}{l}{ }^{\star} P \text { value calculated fo } \\
{ }^{\dagger} P \text { value calculated fo } \\
{ }^{{ }_{1}} 1 \text { patient who died o } \\
\text { Data in parentheses }\end{array}$ & $\begin{array}{l}\text { PET-2 (RIW) vs. } \\
\text { PET-2 (DC) vs. C } \\
\text { pulmonary toxicit } \\
\text { e } 95 \% \text { confidence }\end{array}$ & $\begin{array}{l}\mathrm{T}-2 . \\
-2 . \\
\text { (patient } \\
\text { intervals. }\end{array}$ & ) was excluded & n above & $y s$ & & & \\
\hline
\end{tabular}




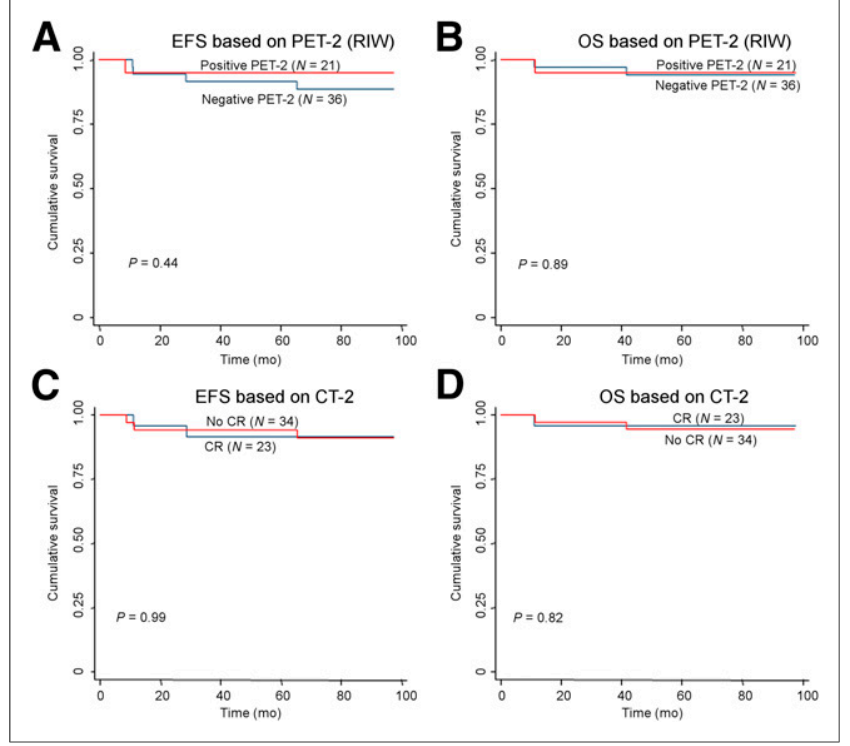

FIGURE 1. Kaplan-Meier graphs for EFS $(A, C)$ and OS (B, D) for interim PET/CT (PET [RIW]-2) (A, B) and interim CECT (CT-2) (C, D). (A) Graph shows EFS according to positive vs. negative scan at interim $\mathrm{PET} / \mathrm{CT}$ based on RIW criteria (PET [RIW]-2). (B) Graph shows OS according to positive vs. negative scan at interim PET/CT based on RIW criteria (PET [RIW]-2). (C) Graph shows EFS according to CR vs. no $\mathrm{CR}$ at interim CECT (CT-2). (D) Graph shows OS according to CR versus no $\mathrm{CR}$ at interim CECT (CT-2).

attained a CR), and 1 had tuberculosis. All the patients who had PR at PET-3 were followed up with repeated imaging and were disease free subsequently.

There was no significant difference in the EFS and OS in patients who had positive PET-3 (RIW) (Figs. 2A and 2B) or residual disease in CT-3 (Figs. 2C and 2D) (Table 5).

\section{Retrospective Analysis Using DC for PET/CT Image Analysis}

DC was used independently by 2 nuclear physicians after retrieval of the scans ( $n=53 / 57$ for interim assessment and 52/ 57 for posttreatment scans). There was substantial agreement between both reviewers in applying the Deauville score. The weighted $\kappa$ for the agreement between the reviewers for the score was 0.58 . However, the agreement for the response assessment based on the score (CR vs. PR) was substantially higher $(\kappa, 0.84)$.

\section{Impact of PET/CT (Deauville) on Specificity, Sensitivity, PPV,} and NPV

At interim assessment, when DC was used, the specificity of PET-2 (Deauville) improved when compared with CT-2 $(91.4 \%$ vs. $42.6 \%, P<0.0001)$. The proportion of patients attaining CR based on DC was also significantly higher in PET-2 (Deauville) than CT-2 (49 [92.4\%] vs. 23 [40.3\%]; $P<0.0001$ ) (Table 2).

In PET-3 (Deauville), the proportion of patients attaining CR (94.2\% vs. $75.4 \%, P=0.007)$ and specificity was significantly better than CT-3 (95.7 vs. 76.4, $P=0.006$ ) (Table 4 ).

\section{Impact of PET/CT (Deauville) on EFS and OS}

The EFS and OS did not differ in patients who were positive or negative by PET-2 (Deauville) (Figs. 3A and 3B; Table 3). The EFS between patients who were positive or negative by PET-3 (Deauville) was statistically insignificant (Fig. 3C); however, the OS between these 2 groups showed a significant difference for poor survival in the group positive by PET-3 (Deauville) $(P=$ 0.029) (Fig. 3D; Table 5).

\section{DISCUSSION}

Our study categorically demonstrated that PET/CT detected more sites than CECT at baseline. Although in this study, the treatment decisions were based on CECT staging, PET/CT staging would have potentially intensified the treatment in $9(15.8 \%)$ patients (one third of the upstaged patients). Our study was not designed to detect the benefit or toxicity based on alteration of treatment based on PET/CT staging. It is, however, worth noting that none of the 9 patients in whom treatment would have been intensified based on baseline PET/CT staging relapsed. Previous studies in pediatric patients have shown similar upstaging in the range of $9 \%-50 \%$ based on PET $(6,7,10,16,17)$. However, all these studies were retrospective, and the impact of treatment modification was not studied in any of them.

In our study, the sensitivity of interim PET/CT was low and a positive PET/CT at interim assessment did not have a significant impact on EFS and OS. However, our study showed that interim

TABLE 3

EFS and OS Based on Interim Assessment*

\begin{tabular}{|c|c|c|c|c|c|c|}
\hline \multirow[b]{2}{*}{ Imaging } & \multicolumn{3}{|c|}{ EFS } & \multicolumn{3}{|c|}{ OS } \\
\hline & Estimate \pm SE & $95 \%$ confidence interval & $P$ & Estimate \pm SE & $95 \%$ confidence interval & $P$ \\
\hline CT-2 $(n=57)$ & & & 0.99 & & & 0.82 \\
\hline $\mathrm{CR}(n=23)$ & $90.6 \pm 4.6$ & $81.4-99.8$ & & $93.7 \pm 3.6$ & $86.5-101$ & \\
\hline No $\mathrm{CR}(n=34)$ & $91.1 \pm 3.6$ & $84-98.2$ & & $93.1 \pm 2.9$ & $85.5-98$ & \\
\hline PET-2 (RIW) $(n=57)$ & & & 0.44 & & & 0.89 \\
\hline Negative (CR) $(n=36)$ & $89.6 \pm 3.8$ & $82.1-97$ & & $93.3 \pm 2.8$ & $87.8-98.7$ & \\
\hline Positive (PR) $(n=21)$ & $93.3 \pm 4.1$ & $85.2-101.3$ & & $93.4 \pm 4.0$ & $85.5-101$ & \\
\hline PET-2 (DC) $(n=53)$ & & & 0.52 & & & 0.61 \\
\hline Negative (CR) $(n=49)$ & $89.6 \pm 3.4$ & $76.7-95.5$ & & 93. \pm 3.4 & $82-97.9$ & \\
\hline Positive (PR) $(n=4)$ & $100^{*}$ & - & & $100^{*}$ & - & \\
\hline
\end{tabular}

${ }^{*}$ There was no relapse in patients with positive PET-2 (DC). 
TABLE 4

Posttreatment Assessment

\begin{tabular}{|c|c|c|c|c|c|c|c|c|}
\hline \multirow[b]{2}{*}{ Parameter } & \multicolumn{2}{|c|}{ CT-3 $(n=55)$} & \multicolumn{2}{|c|}{ PET-3 (RIW) $(n=54)$} & \multirow[b]{2}{*}{$P^{*}$} & \multicolumn{2}{|c|}{ PET-3 (DC) $(n=51)$} & \multirow[b]{2}{*}{$P^{\dagger}$} \\
\hline & No CR & $\mathrm{CR}$ & Positive & Negative & & Positive & Negative & \\
\hline Relapse $(n=4)$ & 1 & 3 & 1 & 3 & & 1 & 3 & \\
\hline Remission $(n=51)^{\ddagger}$ & 12 & 39 & 6 & 44 & & 2 & 45 & \\
\hline Sensitivity (\%) & $25(13.1-78)$ & & $25(13.1-78)$ & & 1 & $25(13.1-78)$ & & 1 \\
\hline Specificity (\%) & $76.4(62.1-86.7)$ & & 88 (74.9-95) & & 0.11 & 95.7 (84.2-99.2) & & 0.006 \\
\hline PPV (\%) & $7.6(0.4-37.9)$ & & $14.2(0.7-57.9)$ & & 0.27 & $33.3(1.7-87.4)$ & & 0.23 \\
\hline NPV (\%) & $92.8(79.4-98)$ & & 93.6 (81.4-98.3) & & 0.86 & $94.1(82.7-98.4)$ & & 0.78 \\
\hline \multicolumn{9}{|c|}{$\begin{array}{l}{ }^{*} P \text { value calculated for } P E T-3(\mathrm{RIW}) \text { vs. CT-3. } \\
{ }^{\dagger} P \text { value calculated for } \mathrm{PET}-3(\mathrm{DC}) \text { vs. CT- } 3 \text {. } \\
\ddagger_{1} \text { patient who died of pulmonary toxicity (patient } 57 \text { ) was excluded from above analysis. } \\
\text { Data in parentheses are } 95 \% \text { confidence intervals. }\end{array}$} \\
\hline
\end{tabular}

PET/CT has significant specificity as compared with conventional imaging. A few retrospective studies have shown high sensitivity of interim PET assessment; however, these studies were limited by the small number of subjects and lack of uniform response assessment criteria $(5,8,10)$.

A prospective study by Furth et al. (10) used the mediastinal blood pool as a reference for PET positivity similar to the RIW criteria used in our study; both these methods are subjective in nature. DC have recently been adopted for pediatric NHL response assessment as well (18). Application of the DC increased the specificity of PET/CT in our cohort. Hence the use of these criteria

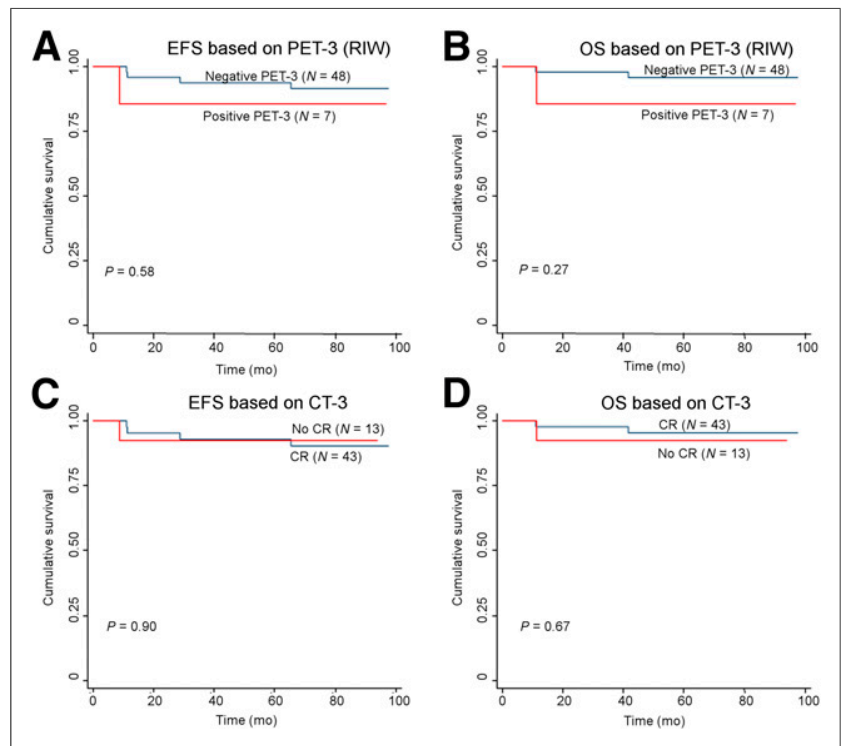

FIGURE 2. Kaplan-Meier graphs for EFS (A, C) and OS (B, D) for posttreatment PET/CT (PET [RIW]-3) (A, B) and posttreatment CECT (CT-3) (C, D). (A) Graph shows EFS according to positive vs. negative scan at posttreatment PET/CT based on RIW criteria (PET [RIW]-3). (B) Graph shows OS according to positive vs. negative scan at posttreatment PET/CT based on RIW criteria (PET [RIW]-3).(C) Graph shows EFS according to CR vs. no CR at posttreatment CECT (CT-3). (D) Graph shows OS according to CR vs. no CR at posttreatment CECT (CT-3). as compared with criteria used in the study by Furth et al. and RIW criteria used in the current study impart objectivity and reproducibility for PET/CT assessment.

The prognostic value of interim PET/CT could not be established in our study. Previous COG (Children's oncology group) study used interim response based on CECT (AHOD0031 trial) for treatment deescalation; it was concluded that in early responders, treatment deescalation could be done without compromising efficacy (19). In this AHOD0031 trial, patients who were slow responders and had interim PET positivity benefitted marginally with treatment escalation. However, in our study 3 of the 4 patients who relapsed had a negative interim PET/CT based on both the criteria (RIW and Deauville). The limitation of the AHOD0031 trial is that the PET positivity was based on reference uptake with mediastinal blood pool, and the study was not powered to address effect of treatment escalation based on PET positivity. Notably in our cohort, based on PET/CT (RIW) interim assessment, we would have potentially escalated treatment in 21 of $57(36.8 \%)$ patients to prevent 1 relapse; based on DC, we would have escalated therapy in 4 of $52(7.6 \%)$ patients and none would have benefitted. Therefore, we do not recommend treatment escalation based on interim PET/CT assessment by either RIW or DC.

Posttreatment evaluation by conventional imaging and PET/CT (RIW) were similar for sensitivity and specificity, and in predicting EFS or OS. However, PET/CT (Deauville) revealed a significantly improved specificity and PPV for PET/CT as compared with conventional CECT imaging. However, a tissue diagnosis is mandatory to rule out an infectious etiology because 1 of 3 patients with positive PET/CT (Deauville) had underlying infection in our cohort. Further, there was a trend toward inferior EFS and a significantly inferior OS in patients who had a positive PET/CT (Deauville) at the end of treatment. Thus, the use of DC improved the PPV and decreased false positivity in comparison to RIW criteria and the criteria used in previous studies $(8,10)$.

The role of histology subtype in interpretation of interim PET/ CT requires further studies because the relative cellular and sclerosis component differs in the histologic subtypes. This could possibly also explain the different findings in the study by Furth et al. when compared with our study. Mixed cellularity subtype and Epstein Barr virus positivity are higher in our population than in the western population $(20,21)$. 
TABLE 5

EFS and OS Based on Posttreatment Assessment

\begin{tabular}{|c|c|c|c|c|c|c|}
\hline \multirow[b]{2}{*}{ Imaging } & \multicolumn{3}{|c|}{ EFS } & \multicolumn{3}{|c|}{ OS } \\
\hline & Estimate \pm SE & $95 \%$ confidence interval & $P$ & Estimate \pm SE & $95 \%$ confidence interval & $P$ \\
\hline CT-3 $(n=56)$ & & & 0.90 & & & 0.67 \\
\hline $\mathrm{CR}(n=43)$ & $91.0 \pm 3.2$ & $84.7-97.3$ & & $94.1 \pm 2.3$ & $89.5-98.8$ & \\
\hline No $\mathrm{CR}(n=13)$ & $87.4 \pm 6.3$ & $75-99.8$ & & $87.6 \pm 6.1$ & $75.6-99.6$ & \\
\hline PET-3 (RIW) $(n=55)$ & & & 0.58 & & & 0.27 \\
\hline Negative (CR) $(n=48)$ & $91.7 \pm 2.9$ & $86-97.4$ & & $94.5 \pm 2.1$ & $90.3-98.7$ & \\
\hline Positive (PR/PD) $(n=7)$ & $84.1 \pm 11.6$ & $61-107$ & & $84.5 \pm 11.3$ & $62.3-106.7$ & \\
\hline PET-3 (DC) $(n=52)$ & & & 0.094 & & & 0.029 \\
\hline Negative (CR) $(n=49)$ & $91.8 \pm 2.8$ & $86.2-97.4$ & & $94.5 \pm 2.0$ & $90.5-98.6$ & \\
\hline Positive (PR/PD) $(n=3)$ & $65.5 \pm 23.2$ & $20-111.1$ & & $66.4 \pm 22.5$ & $22.2-110$ & \\
\hline
\end{tabular}

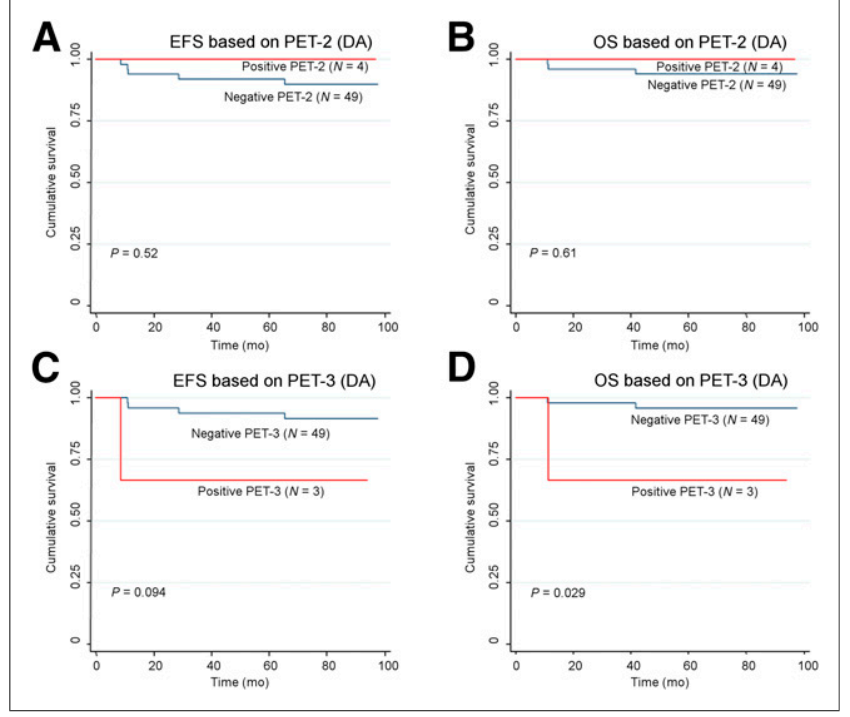

FIGURE 3. Kaplan-Meier graphs for EFS (A, C) and OS (B, D) for interim PET/CT (PET [Deauville]-2) (A, B) and posttreatment PET/CT based on DC (PET [Deauville]-3) (C, D). (A) Graph shows EFS according to positive vs. negative scan at interim PET/CT based on DC (PET [Deauville]-2). (B) Graph shows OS according to positive vs. negative scan at interim PET/CT based on DC (PET [Deauville]-2). (C) Graph shows EFS according to positive vs. negative scan at posttreatment PET/CT based on Deauville criteria (PET [Deauville]-3). (D) Graph shows OS according to positive vs. negative scan at posttreatment PET/CT based on DC (PET [Deauville]-3).

The major strength of our study is adequate follow-up duration, direct comparison of CECT and PET/CT modalities by both RIW and DC, and PET/CT response assessment by 2 experienced nuclear medicine specialists who had good agreement on assessment. Further studies based on DC for PET/CT interpretation can bring uniformity for trial comparisons and to identify patients with high risk of relapse.

\section{CONCLUSION}

Baseline imaging with PET/CT may potentially affect the treatment decision, which may not prevent relapse or prolong survival. So, further studies to evaluate the role of baseline imaging with PET/CT are needed. Our study shows that there is no conclusive evidence for PET-based risk stratification for escalation or deescalation of therapy. Escalation of therapy based on interim PET in pediatric HL needs further conclusive evidence to justify its use; this is even more relevant in HL as compared with other malignancies because most patients can be salvaged at relapse. Posttreatment PET/CT assessment by DC is recommended; however, any treatment decision based on PET/CT assessment needs to be confirmed with a tissue diagnosis.

\section{DISCLOSURE}

Funding was received from the Department of Biotechnology, Government of India. No other potential conflict of interest relevant to this article was reported.

\section{REFERENCES}

1. Mauz-Körholz C, Metzger ML, Kelly KM, et al. Pediatric Hodgkin lymphoma. J Clin Oncol. 2015;33:2975-2985.

2. Gallamini A, Hutchings M, Rigacci L, et al. Early interim 2-[18f]fluoro-2deoxy-d-glucose positron emission tomography is prognostically superior to international prognostic score in advanced-stage Hodgkin's lymphoma: a report from a joint Italian-Danish study. J Clin Oncol. 2007;25: 3746-3752.

3. Gallamini A, Barrington SF, Biggi A, et al. The predictive role of interim positron emission tomography for Hodgkin lymphoma treatment outcome is confirmed using the interpretation criteria of the Deauville five-point scale. Haematologica. 2014;99:1107-1113.

4. Oki Y, Chuang H, Chasen B, et al. The prognostic value of interim positron emission tomography scan in patients with classical Hodgkin lymphoma. $\mathrm{Br}$ J Haematol. 2014;165:112-116.

5. Lopci E, Burnelli R, Ambrosini V, et al. ${ }^{18}$ F-FDG PET in pediatric lymphomas: a comparison with conventional imaging. Cancer Biother Radiopharm. 2008;23: 681-690.

6. Paulino AC, Margolin J, Dreyer Z, Teh BS, Chiang S. Impact of PET-CT on involved field radiotherapy design for pediatric Hodgkin lymphoma. Pediatr Blood Cancer. 2012;58:860-864.

7. Miller E, Metser U, Avrahami G, et al. Role of ${ }^{18} \mathrm{~F}-\mathrm{FDG}$ PET/CT in staging and follow-up of lymphoma in pediatric and young adult patients. J Comput Assist Tomogr. 2006;30:689-694.

8. Levine JM, Weiner M, Kelly KM. Routine use of PET scans after completion of therapy in pediatric Hodgkin disease results in a high false positive rate. J Pediatr Hematol Oncol. 2006;28:711-714. 
9. Cheng G, Servaes S, Zhuang H. Value of ${ }^{18}$ F-fluoro-2-deoxy-D-glucose positron emission tomography/computed tomography scan versus diagnostic contrast computed tomography in initial staging of pediatric patients with lymphoma. Leuk Lymphoma. 2013;54:737-742.

10. Furth C, Steffen IG, Amthauer H, et al. Early and late therapy response assessment with $\left[{ }^{18} \mathrm{~F}\right]$ fluorodeoxyglucose positron emission tomography in pediatric Hodgkin's lymphoma: analysis of a prospective multicenter trial. J Clin Oncol. 2009;27:4385-4391.

11. Ilivitzki A, Radan L, Ben-Arush M, Israel O, Ben-Barak A. Early interim FDG PET/CT prediction of treatment response and prognosis in pediatric Hodgkin disease: added value of low-dose CT. Pediatr Radiol. 2013;43:86-92.

12. Lister TA, Crowther D, Sutcliffe SB, et al. Report of a committee convened to discuss the evaluation and staging of patients with Hodgkin's disease: Cotswolds meeting. J Clin Oncol. 1989;7:1630-1636.

13. Fryer CJ, Hutchinson RJ, Krailo M, et al. Efficacy and toxicity of 12 courses of ABVD chemotherapy followed by low-dose regional radiation in advanced Hodgkin's disease in children: a report from the Children's Cancer Study Group. J Clin Oncol. 1990;8:1971-1980.

14. Cheson BD, Pfistner B, Juweid ME, et al. Revised response criteria for malignant lymphoma. J Clin Oncol. 2007;25:579-586.
15. Barrington SF, Qian W, Somer EJ, et al. Concordance between four European centres of PET reporting criteria designed for use in multicentre trials in Hodgkin lymphoma. Eur J Nucl Med Mol Imaging. 2010;37:1824-1833.

16. Montravers F, McNamara D, Landman-Parker J, et al. $\left[{ }^{18} \mathrm{~F}\right] \mathrm{FDG}$ in childhood lymphoma: clinical utility and impact on management. Eur J Nucl Med Mol Imaging. 2002;29:1155-1165.

17. Depas G, Barsy CD, Jerusalem G, et al. ${ }^{18}$ F-FDG PET in children with lymphomas. Eur J Nucl Med Mol Imaging. 2005;32:31-38.

18. Sandlund JT, Guillerman RP, Perkins SL, et al. International pediatric non-Hodgkin lymphoma response criteria. J Clin Oncol. 2015;33:2106-2111.

19. Friedman DL, Chen L, Wolden S, et al. Dose-intensive response-based chemotherapy and radiation therapy for children and adolescents with newly diagnosed intermediate-risk Hodgkin lymphoma: a report from the Children's Oncology Group Study AHOD0031. J Clin Oncol. 2014;32:36513658.

20. Dinand V, Arya LS. Epidemiology of childhood Hodgkins disease: is it different in developing countries? Indian Pediatr. 2006;43:141-147.

21. Arya LS, Dinand V, Thavaraj V, et al. Hodgkin's disease in Indian children: outcome with chemotherapy alone. Pediatr Blood Cancer. 2006;46: 26-34. 